\title{
How to Solve the Problem of Chronic Oxidative Stress?
}

\section{Velio Bocci and Emma Borrelli*}

Department of Chemistry, Pharmacy and Biotechnologies, University of Siena, Italy

\begin{abstract}
Cardiovascular and related chronic diseases (CVD), macula degeneration (MD), chronic obstructive pulmonary diseases (COPD), type II diabetes and brain degenerative diseases are due to an initial inflammation later complicated by a chronic oxidative stress (COS).

They are the main cause of disability and mortality because orthodox drugs, although effective, hardly reduce the COS. The integration of ozonated autohemotherapy (AHT), by the activation of a transcription factor called Nrf2, is able to reactivate the innate antioxidant system and re-establish homeostasis.
\end{abstract}

Keywords: Chronic oxidative stress; Ozone therapy; Cysteine code; Hinge; Latch model

The possibility of preventing and treating cardiovascular diseases and several other chronic diseases, thus reducing inability and early death is not only a problem of affluent countries but is reaching epic proportions in less developed continents as Asia and South-America due to high-fat, heavily salted diet, reduced physical activity, heavy consumption of alcohol and tobacco and poor or absent medical attention. Worldwide more than 1.4 billion adults are overweight and 500 million are obese. CVD including stroke with chronic respiratory diseases, type II diabetes, macular degeneration in 2011 have been responsible in the world of 21.8 millions deaths, opposed to about six millions deaths due to HIV-AIDS, diarrheal diseases, road injury and prematurity [1]. Abuse of alcohol and other drugs kill more people than AIDS and tuberculosis combined. Consequently, besides improving medical treatments in poor countries, it appears indispensable to integrate orthodox medications by tackling the obesity epidemic, promoting healthier diets and reducing the use of alcohol and tobacco. However this is not enough because the official orthodox treatments of statins, anti-hypertensive, anti-aggregant drugs and antidiabetics, although very useful, are not able to correct the chronic oxidative stress that ultimately is the cause of death.

\section{How to Solve this Problem}

During the last two decades several approaches, such as the administration of antioxidants, the value of calorie restriction (CR), and of a daily exercise stress, the administration of selected compounds or of oxygen-ozonetherapy have been experimented and, at different degrees, they may be able to progressively reduce the chronic oxidative stress and restore a normal redox system [2]. Several clinical trials experimenting the use of a variety of antioxidants such as ascorbic acid or glutathione (GSH) or a complex of vitamins on the whole have been disappointing because either they cannot enter into the cells or they act aspecifically, thus unable to restore homeostasis. On the other hand equilibrated calorie restriction associated to a daily moderate exercise stress appears useful but not everyone accept this approach for a long period [3]. More recently the daily administration of both curcumin and L-sulforaphane has shown some advantages but their bioavailability remains to be improved [4]. Oxygen-ozonetherapy, if performed correctly and for a long period of time, appears effective but it must follow precise rules. This method was firstly experimented (1975) by a German physician, Hans Wolff (1908-1981) and in Germany some 15,000 physicians performed it daily: Unfortunately the methodology has remained empirical in many European countries, 13 States of USA,
Canada, South America, India, China and Japan. However today, by using a precise schedule and by using a modern ozone generator able to yield a very precise ozone dose, the potential ozone toxicity is nil.

Ozone is a very reactive gas and its electrochemical potential value is: $\mathrm{E}^{\circ}=+2.076 \mathrm{~V}$. It is well known that anthropogenic ozone associated with fine particulate matter present in urban and rural regions of the USA contribute to global premature mortality [5]. Therefore ozone ought to be never inhaled by anybody. Luckily ozone, added in minimal amounts to human blood ex vivo, represents a tolerable stress because its reactivity is fully neutralized by the wealth of antioxidants (ascorbic acid, uric acid, albumin and GSH) and by the polyunsaturated fatty acids (PUFA) present in human blood. Therefore in 2006 it was demonstrated that the small ozone dosage used in patients is not toxic [6]. First of all ozone is added to $100-150 \mathrm{ml}$ of human blood withdrawn in a sterile vacuum glass flask in the same respective volume. The variable blood volume is related to a variable body weight ranging from 50 to $100 \mathrm{~kg}$. Normally the treatment is bi-weekly and during the first week, the ozone concentration is of $10 \mu \mathrm{g} / \mathrm{ml}$. In other words for $100 \mathrm{ml}$ of blood, only 1 $\mathrm{mg}$ of ozone is necessary. Within 1-2 min ozone, by completely reacting with the wealth of antioxidants present in plasma and with the PUFA, is no longer present. During the second week, the ozone concentration may be raised of $5 \mu \mathrm{g} / \mathrm{ml}$ with a dose of $15 \mu \mathrm{g} / \mathrm{ml}$. By increasing $5 \mu \mathrm{g} / \mathrm{ml}$ every successive weeks, by the fifth week, we may add $30 \mu \mathrm{g} / \mathrm{ml}$ of ozone per $\mathrm{ml}$ of blood or, in other words, $100 \mathrm{ml}$ of blood will accept an ozone dose of $3 \mathrm{mg}$. These progressive concentrations are absolutely safe and represent a well tolerated stress for any patient [7]. This scheme obeys to the hormetic mechanisms to be pharmacologically active but atoxic. After $5 \mathrm{~min}$, the oxygenated-ozonated blood is infuse into the donor patient and only the auto-transfusion is allowed.

Chemically the reaction of ozone with blood follows:

Antioxidants +PUFA (-R-CH=CH-R-) $+\mathrm{H}_{2} \mathrm{O}+\mathrm{O}_{3} \rightarrow$ partly

*Corresponding author: Emma Borrelli, Department of Medical Biotechnologies University of Siena, Italy, Tel: +390577585731 ; Fax: +390577585731 ; E-mail ebitaly2007@libero.it

Received June 02, 2015; Accepted June 23, 2015; Published June 29, 2015

Citation: Bocci V, Borrelli E (2015) How to Solve the Problem of Chronic Oxidative Stress?. Bioenergetics 4: 123. doi:10.4172/2167-7662.1000123

Copyright: $\odot 2015$ Bocci V, et al. This is an open-access article distributed unde the terms of the Creative Commons Attribution License, which permits unrestricted use, distribution, and reproduction in any medium, provided the original author and source are credited. 
oxidized antioxidants $+\mathrm{H}_{2} \mathrm{O}_{2}+2 \mathrm{R}-\mathrm{CHO}$.

Thus ozone acts as a pro-drug and allows the generation of physiological constituents such as hydrogen peroxide and aldehydes as terminal products of the lipid peroxidation. It must be mentioned that the partly oxidized antioxidants are reduced within $15 \mathrm{~min}$ to their active states by the activity of electron donation by $\mathrm{NADPH}$, vitamin $\mathrm{E}$ and thioredoxin.

Consequently the ozone reaction has generated two important messengers, which, by infusing the ozonated blood into the donor, are responsible for the successive pharmacological effects.

\section{What is the fate of $\mathrm{H}_{2} \mathrm{O}_{2}$ and alchenals as aldehyde products?}

a) $\mathrm{H}_{2} \mathrm{O}_{2}$ is a common compound in blood cells and, by entering into the mass of erythrocytes activates glycolysis with a transient increase of ATP and of 2-3-diphosphoglycerate (2,3-DPG). This compound causes a shift to the right of oxyhemoglobin and this very useful process improves the release of oxygen into ischemic tissues, an important step in ischemic diseases.

b) The oxidized PUFA generates the terminal compound which is an aldehyde with only 9 carbons called 4 -hydroxynonenal. This is partly catabolised via the bile or urine but some is bound to either the $-\mathrm{SH}$ group of cysteine 34 of albumin or to the cysteine or to GSH.

\section{What is the Function of the 4-Hydroxynonenal?}

The residual alchenal has the critical duty to activate the innate antioxidant system of the body. The compound, via the circulation of blood, is transported into many cells of the body including brain and this is its most important function. Patients afflicted by the chronic oxidative stress contains cells unable to activate their antioxidant system thus undergoing death. However their cytoplasm contains a transcription factor denominated Nuclear related factor 2 (Nrf2) that needs to be activated. The Nrf2 protein is unable to act because it is bound to a repressive protein called Keap1 (Kelch-like erythroid cellderived protein), which is an adaptor protein for a Cullin 3 (Cul3)dependent ubiquitination and degradation of Nrf2: Consequently the complex Nrf2-Keap1 remains unable to act and is going to enter the proteasome every about $20 \mathrm{~min}$ to be digested. Thus the cell, depressed by an excess of oxidants due to the chronic inflammation is unable to help the afflicted organism. However the protein Keap1 contains many cysteines and particularly Cys 273 and Cys 288 are critical for Keap1 function. Luckily the 4-hydroxynonenal transported by the blood is able to enter the cell and bind to either Cys 273 or Cys 288 . The new binding renders Keap 1 unable to direct the ubiquitination of the complex and allows the release of the Nrf2 protein: This step is fundamental in preventing the exacerbations of stress-induced diseases, such as cardiovascular diseases, COPD, diabetic nephropathy, multiple sclerosis and probably malaria: almost needless to say that there other Nrf2 activators binding to other cysteines of Keap1 like triterpenoids, bardoxolone snd dimethyl fumarate but obviously the use of ozonetherapy represent a great advantage to be atoxic, effective and readily available. The free Nrf2, which is a protein of 90-110 molecular weight, is then able to leave the cell cytoplasm and move into the cell nucleus while, after binding a Maf protein, can activate the antioxidant response element (ARE) and can induce about 230 genes belonging to the phase II antioxidant and detoxification response. The induced genes include the synthesis of GSH, GSH reductase, GSH transferase, GSH peroxidases, thioredoxin, thioredoxin reductase, NADPH, Nad(P)- quinone oxidoreductase 1 (NQO1), UDP syaliltransferase and hemeoxygenase 1 able to increase the level of $\mathrm{CO}$ and bilirubin. Nrf2 also plays a role in the negative regulation of insulin and growth hormone which increases stress resistance [8].

It remains doubtful if the activation of Nrf2 is really useful in metastatic cancer because cancer cells become chemotherapy-resistant. Moreover Nrf2 proteins and mRNA levels decline within 24 hours and therefore two or at most three sessions weekly are enough. It was already mentioned that either an appropriate dietary restriction or a daily use of curcumin and sulforaphane exert a benefit but it will be necessary to increase their bioavailability and dosages to become of practical use. On the other hand ozonetherapy is frequently carried out free of charge and it is certainly effective.

\section{Conclusions}

It must be clarified that the activation of $\mathrm{Nrf} 2$ must be performed in diseases such as cardiovascular including stroke, COPD, age-related macular degeneration (dry form only), typeII diabetes. Moreover clinical studies must be performed in degenerative brain diseases where the chronic oxidative stress ought to be eliminated. Evidence of great improvement has been already documented in chronic limb ischemia, macular degeneration and in COPD [9,10]. Patients reacquire a wellfeeling and can work normally. Obviously it would be necessary to perform now very large trials on these diseases and this paper possibly will encourage other clinicians. Orthodox medicines are effective but, except statins,cannot solve the problem of the chronic oxidative stress and the need of integration with ozonetherapy appears essential.

\section{References}

1. World Health Organisation Report, 2011.

2. Bocci V, Borrelli E (2015) A practical approach for restoring homeostasis in diseases characterized by a chronic oxidative stress. J Adv Med Pharm Sci 2: $135-143$.

3. Holloszy JO, Fontana (2007) Caloric restriction in humans. Exper Geront 42 709-712.

4. Aggarwal BB, Harikumar KB (2009) Potential therapeutic effects of curcumin the antinflammatory agent, against neurodegenerative, cardiovascular, pulmonary, metabolic, autoimmune and neoplastic diseases. Int J Biochem Cell Biol 41: 40-59.

5. Bocci V (2011) Ozone A new medical drug. Second Edition, Springer Verlag, Dordrecht, Netherlands 1-400.

6. Borrelli E, Bocci V (2010) Basic, biologic, therapeutic effects of ozone therapy in human medicine. Encyclopedia of Life Support Systems, Eolss Publisher, Oxford UK.

7. Bocci V (2006) Scientific and medical aspects of ozone therapy. State of the art. Arch Med Res 37: 425-435

8. Pecorelli A, Bocci V, Acquaviva A (2013) Nrf2 activation is involved in ozonated human serum upregulation of $\mathrm{HO} 1$ in endothelial cells. Toxicol Appl Pharmacol 267: $30-40$.

9. Borrelli E, Bocci V (2014) Oxygen ozone therapy in the treatment of chronic obstructive pulmonary disease: an integrative approach. Am J Clin Exper Med 2: $9-13$.

10. Borrelli E, Diadori A, Zalaffi A, Bocci V (2012) Effect of major ozonated autohaemotherapy in the treatment of dry age related macular degeneration: a randomized controlled clinical study.Int J Ophtalmol 5: 708-712. 